Pesq. Vet. Bras. 36(3):221-226, março 2016 DOI: $10.1590 / \mathrm{S} 0100-736 \mathrm{X} 2016000300012$

\title{
Anatomia e histologia do trato urinário do macaco-prego (Sapajus apella) $^{1}$
}

\author{
Ana R. Lima²*, Sheyla B. Guimarães ${ }^{2}$, Érika Branco ${ }^{3}$, Elane G. Giese ${ }^{3}$, \\ José A.P.C. Muniz ${ }^{4}$, Rose Eli G. Ricci ${ }^{5}$ e Maria A. Miglino ${ }^{5}$
}

\begin{abstract}
Lima A.R., Guimarães S.B., Branco E., Giese E.G., Muniz J.A.P.C., Ricci R.E.G. \& Miglino M.A. 2016. [Anatomy and histology of the urinary tract in the capuchin monkey (Sapajus apella).] Anatomia e histologia do trato urinário do macaco-prego (Sapajus apella). Pesquisa Veterinária Brasileira 36(3):221-226. Laboratório de Pesquisa Morfológica Animal, Faculdade de Medicina Veterinária, Universidade Federal Rural da Amazônia, Av. Pres. Tancredo Neves 2501, Montese, Belém, PA 66077-530, Brazil. E-mail: vetlima@uol.com.br

Sapajus apella is a primate of the New World of midsize and with geographical distribution from South America to Argentina. Good models for the study and in recent years have been widely used for this purpose. The progressive destruction of the natural habitat of these animals has led to migrate to other regions, thus making them more susceptible to hunting predator. The need for the preservation of wild species arouses interest by a greater anatomic and clinical knowledge of these animals. The present study was aimed to examine and describe the morphological aspects, as far as the macroscopic and microscopic anatomy of the organs of the urinary system of female Sapajus apella aiming to extend the anatomical knowledge for this species. Four females were used, from the National Center of Primates in Ananindeua - PA. The urinary system of S. apella has similar characteristics to other primates both human and non-human and domestic animals. The kidneys showed macroscopic aspects similar to the kidneys of humans, but with the same classification of other non-human primates and some domestic animals. The bladder was similar to domestic animals and primates in general, human and non-human. The same occurred when we analyze these components histologically.

INDEX TERMS: Capuchin monkey, Sapajus apella, urinary system, macroscopic anatomy, microscopic anatomy.
\end{abstract}

RESUMO.- Sapajus apella é um primata do Novo Mundo de porte médio e com distribuição geográfica desde a América do Sul até a Argentina. São bons modelos para estudo e

\footnotetext{
${ }^{1}$ Recebido em 12 de janeiro de 2015.

Aceito para publicação em 29 de novembro de 2015.

${ }^{2}$ Laboratório de Pesquisa Morfológica Animal (LaPMA), Faculdade de Medicina Veterinária, Universidade Federal Rural da Amazônia (UFRA), Avenida Presidente Tancredo Neves 2501, Montese, Belém, PA 66077530, Brasil. E-mails: sheylavet@ibest.com.br; *Autor para correspondência: vetlima@uol.com.br

${ }^{3}$ Instituto de Saúde e Produção Animal (ISPA), Faculdade de Medicina Veterinária, Universidade Federal Rural da Amazônia (UFRA), Av. Presidente Tancredo Neves 2501, Montese, Belém, PA 66077-530, Brasil. E-mails: ebranco.ufra@gmail.com, elaguerreiro@uol.com.br

${ }^{4}$ Centro Nacional de Primatas (Cenp), Instituto Evandro Chagas (IEC), Rodovia BR-316 Km 7, Ananindeua, PA 67030-000, Brasil. E-mail: japcmuniz@hotmail.com

${ }^{5}$ Faculdade de Medicina Veterinária e Zootecnia (FMVZ), Universidade de São Paulo (USP), Av. Prof. Dr. Orlando Marques de Paiva 87, Cidade Universitária, São Paulo, SP 05508-270, Brasil. E-mails: ml.salvadori@uol. com.br, roseeli@usp.br,miglino@usp.br
}

nos últimos anos vêm sendo amplamente utilizados para este fim. A destruição progressiva do habitat natural desses animais os tem levado a migrarem para outras regiões, tornando-os, assim, mais susceptíveis à caça predatória. A necessidade de preservação das espécies silvestres desperta interesse por um maior conhecimento anatômico e clínico para estes animais. 0 presente estudo teve como objetivo examinar e descrever os aspectos morfológicos, quanto a anatomia macroscópica e microscópica dos órgãos do sistema urinário de fêmeas da espécie Sapajus apella visando estender o conhecimento anatômico para esta espécie. Foram utilizadas quatro fêmeas, oriundas do Centro Nacional de Primatas de Ananindeua/PA. istema urinário de S. apella apresenta características similares a outros primatas tanto humanos quanto não humanos e aos animais domésticos. Os rins apresentaram aspectos macroscópicos semelhantes aos rins de humanos, mas com a mesma classificação de outros primatas não humanos e alguns animais domésticos. A bexiga assemelhou-se aos animais domésticos e 
aos primatas de maneira geral, humano e não humano. 0 mesmo ocorreu quando analisamos estes órgãos histologicamente.

TERMOS DE INDEXAÇÃO: Macaco-prego, Sapajus apella, sistema urinário, anatomia macroscópica, anatomia microscópica.

\section{INTRODUÇÃO}

Descrições recentes realizadas por Lynch, Silva \& Rylands (2012) e Lynch et al. (2011), demonstraram que há duas ramificações para macacos-pregos com origens geográficas separadas. 0 estudo foi baseado em evidências moleculares, biogeográficas e diferenças na morfologia, ecologia e comportamento. 0 que levou a separação destes primatas em dois gêneros; Sapajus (Kerr, 1792), para macacos-prego mais robustos (muitos deles apresentam "topete" na cabeça) e Cebus (Erxleben, 1777), para macacos-prego mais esguios. Por este motivo, no presente estudo ocorre em diferentes momentos a citação da seguinte forma: Sapajus (Cebus) apella pois não podemos alterar o que já foi publicado por outros autores.

Os Sapajus (Cebus) apella são primatas do gênero Cebus, família Cebidae, sendo classificados como primatas do Novo Mundo. São animais de porte médio com distribuição geográfica desde a América do Sul até a Argentina, habitando principalmente a floresta tropical amazônica e atlântica no Brasil (Guerim 2001). Os macacos do gênero Cebus apresentam características que os tornam, do ponto de vista etológico bons modelos para estudo como: longa expectativa de vida (infância e juventude prolongada); alta taxa de encefalização; grande tendência à exploração e manipulação; partilha de alimentos; dieta onívora; além de comportamentos sociais complexos como cooperação e formação de coalisões (Fragaszy et al. 2004).

Estes animais são onívoros e a maior parte de sua dieta é constituída de frutos e uma pequena quantidade de insetos (Wilson \& Reeder 1992, Auricchio 1995). 0 gênero apresenta uma ampla área de ocorrência, desde o norte da Colômbia até o Sul da Argentina, desde a Planície Litorânea Atlântica até a Cordilheira dos Andes, distribuindo-se geograficamente por quase todo Brasil, principalmente em florestas tropicais (Bolina et al. 2005). Difundido tanto ao norte quanto ao sul da Amazônia Legal Brasileira e no Cerrado, locais que sofrem rotineiramente intensa intervenção humana. A destruição progressiva do habitat natural desses animais os tem levado a migrarem para outras regiões, tornando-os, assim, mais susceptíveis à caça predatória (Alves et al. 2007).

A necessidade de preservação das espécies silvestres desperta interesse por um maior conhecimento anatômico e clínico para estes animais (Alves et al. 2007, Teixeira et al. 2015). Segundo Ribeiro (2002) o conhecimento da anatomia de qualquer animal silvestre é de fundamental importância para sua preservação e proteção.

O sistema urinário compreende um par de rins que produzem a urina a partir do sangue; os ureteres que conduzem a urina dos rins; a bexiga, onde a urina fica armazenada até que possa ser liberada; e a uretra, pela qual finalmente ela passa para o exterior (Dyce et al. 2004).
0 presente estudo tem como objetivo descrever os aspectos morfológicos, quanto a anatomia macroscópica e microscópica do sistema urinário de fêmeas do gênero Sapajus apella, assim como mensurá-los, visando fornecer dados para o conhecimento desta espécie e, auxiliar biotérios quanto a existência de problemas quanto a este sistema.

\section{MATERIAL E MÉTODOS}

Para o desenvolvimento da presente pesquisa, foram utilizadas quatro fêmeas: duas jovens e duas adultas de Sapajus apella, oriundas do Centro Nacional de Primatas de Ananindeua/PA com aprovação no Comitê de Ética para uso de Animais (CEPAN/IEC/ SVS/MS) no 008/2010.

Os animais tiveram o sistema circulatório preenchido com látex Neoprene 450 para evidencialização das artérias e posteriormente foram fixados em solução aquosa de formaldeído a $10 \%$ por meio de injeções intramusculares, subcutâneas e intracavitárias sendo mantidos nesta solução por no mínimo $72 \mathrm{~h}$ para posterior dissecação. Tal procedimento foi realizado pois os animais estão sendo utilizados em outras pesquisas.

Toda a nomenclatura adotada foi baseada na Nomina Anatômica Veterinária (International Committee on Veterinary Gross Anatomical Nomenclature 2012).

Para acessar os órgãos de interesse foi realizada uma incisão na linha mediana ventral para abertura da cavidade abdominal e pélvica, permitindo a remoção dos órgãos urinários.

Para a análise microscópica as amostras dos órgãos foram processadas com o uso de técnicas de rotina e coradas com Hematoxilina e Eosina e Tricromo de Masson, sendo os cortes analisados em microscópios ópticos modelos Axio Imager Z2 (Zeiss ${ }^{\circledR}$ ) e Eclipse E-800 (Nikon ${ }^{\circledR}$ ).

Amostras dos rins foram submetidas à microscopia eletrônica de varredura as quais foram fixadas por imersão em solução de Karnovsky modificada contendo glutaraldeído 2,5\% e paraformaldeído $2 \%$ em tampão fosfato de sódio 0,1 M (pH 7,2), de acordo com Watanabe \& Yamada (1983) e seguindo o protocolo de Murakami (1974). Sendo analisadas com o auxílio de microscópio eletrônico de varredura (LEO 435VP).

\section{RESULTADOS}

Macroscopicamente observamos a presença dos rins, localizados no espaço retroperitoneal, com seus respectivos ureteres desembocando na bexiga urinária.

Os rins de Sapajus apella não estavam dispostos simetricamente e mostraram formatos diferentes, o rim direito era semelhante a um grão de feijão e o esquerdo apresentou a extremidade inferior afilada e a superior arredondada (Fig.1). Externamente os rins encontraram-se recobertos por uma cápsula fibrosa e a extremidade superior apresentou sintopia com a glândula adrenal. Possuíam duas faces: uma côncava (hilo renal), onde verificou-se a presença de artéria e veia renal e ureter, e a outra face convexa em contato com a parede abdominal. Por meio de um corte longitudinal do rim pode-se observar que internamente este é composto por uma região cortical bastante vascularizada e uma medular com presença de pelve renal (Fig.1).

Microscopicamente confirmou-se a divisão do parênquima renal em córtex e medula. Sendo que, a principal característica do córtex renal é a presença de corpúsculos renais, estruturas esféricas constituídas pelo glomérulo, que aparece como uma grande massa celular, circundada 

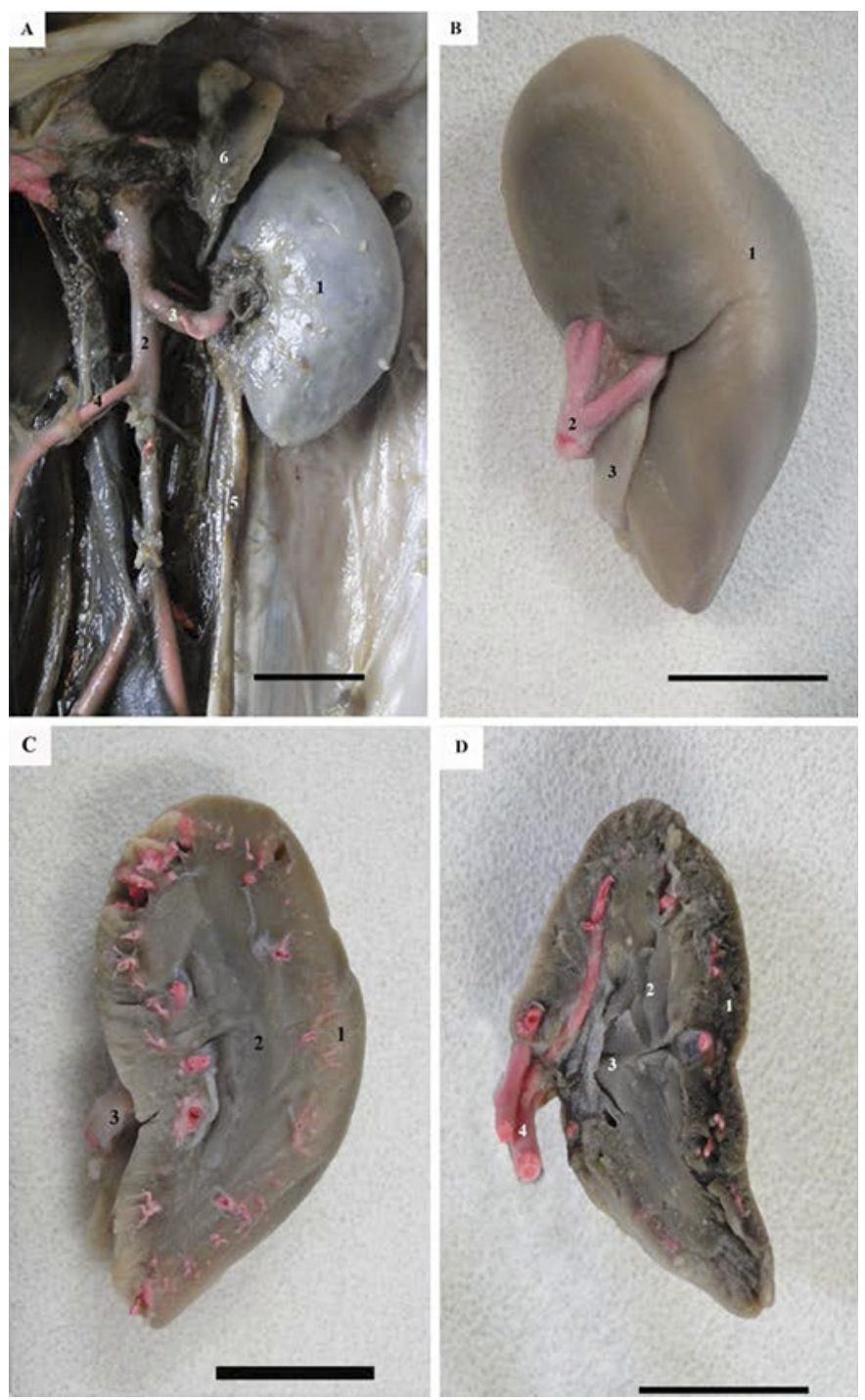

Fig.1. Os rins de Sapajus apela. (A) Topografia do rim esquerdo: rim (2), aorta (2), artéria renal (3), artéria mesentérica cranial (4), ureter (5), adrenal esquerda (6). (B) Rim direito (1), artéria renal (2), ureter (3). (C) Corte longitudinal do rim: córtex renal (1), medula renal (2), ureter (3). (D) Corte longitudinal do rim: córtex renal (1), medula renal (2), pelve renal (3), artéria renal (4). Barra: $1 \mathrm{~cm}$.

pela cápsula de Bowman e túbulos contorcidos proximal e distal estruturas também visualizadas pela microscopia eletrônica de varredura (Fig.2).

0 ureter dos exemplares de Sapajus apella analisados apresentou-se como um ducto de comunicação entre os rins e a bexiga, originando-se na pelve renal de cada um dos rins e seguindo um trajeto sagital contra o teto do abdome (Fig.1) até alcançar o ligamento largo do útero onde inclina-se medialmente. Seguindo nesta posição até sua desembocadura, por meio dos óstios ureterais externos, na superfície dorsal da bexiga urinária próximo ao colo na região do trígono vesical. Microscopicamente foi possível observar o epitélio de transição, tecido conjuntivo e músculo liso (Fig.2D).

A bexiga urinária do Sapajus apella está localizada na região pélvica sob o aparelho reprodutor feminino, como uma estrutura globosa que recobre a vagina, apresentando vértice, seguido do corpo e caudalmente o colo (Fig.3A).

Microscopicamente a bexiga de Sapajus apella apresentou uma túnica mucosa, túnica muscular e serosa (Fig.3B). A túnica mucosa apresenta-se pregueada e é composta por tecido conjuntivo e revestida por um epitélio de transição (Fig.3C). A túnica muscular é constituída por músculo liso organizado em três camadas uma longitudinal interna, uma circular média e uma longitudinal externa. A túnica serosa é constituída por uma camada de epitélio que repousa sobre uma pequena quantidade de tecido conjunto (Fig.3B). Analisando os cortes histológicos da bexiga urinária foi
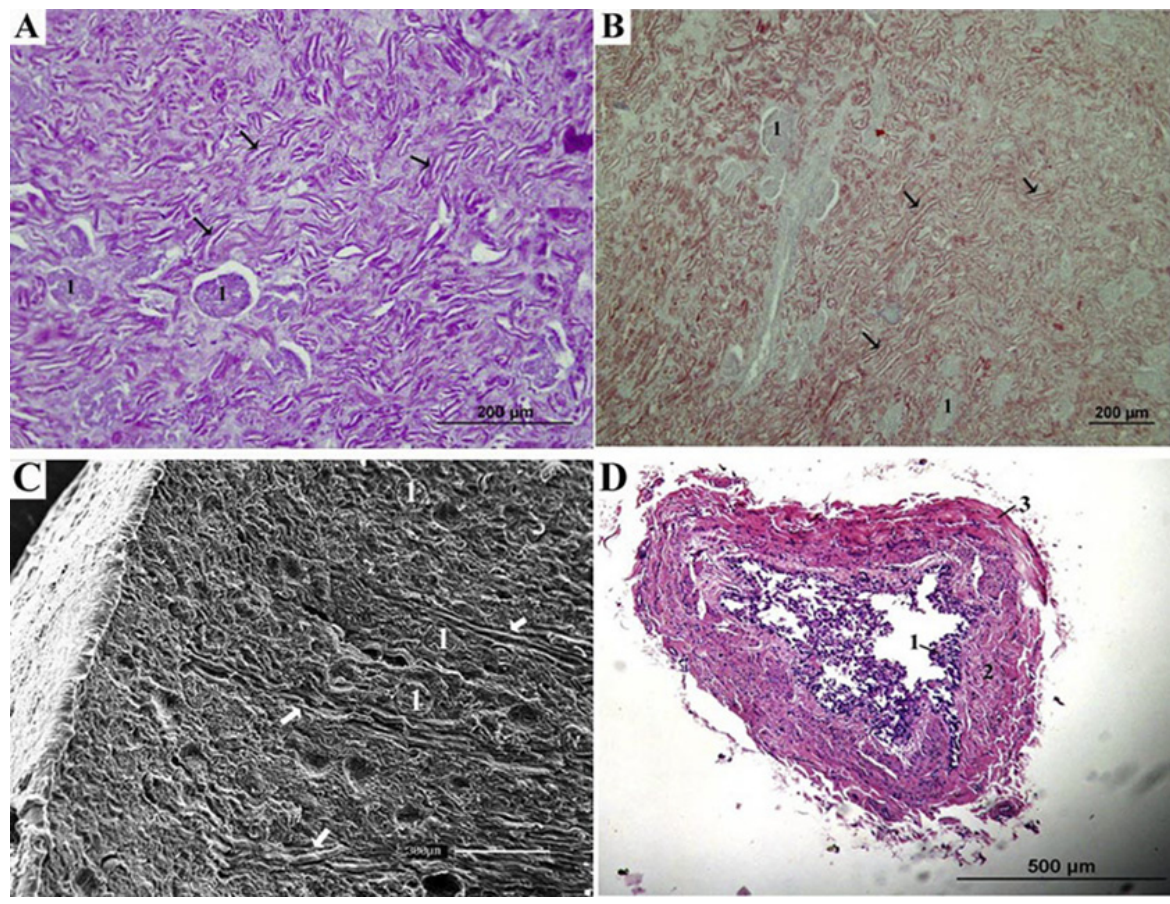

Fig.2. (A,B) Rim de Sapajus apela. (A) Córtex renal com corpúsculos renais (1) e túbulos contornados (setas). Hematoxilina-Eosina. (B) Ttúbulos contornados (setas) e corpúsculos renais (1). Tricrômio de Masson, Barra: $200 \mu \mathrm{m}$. (C) Eletromicrografia de varredura onde observam-se a presença de glomérulos (1) e túbulos contornados (setas). Barra: $300 \mu \mathrm{m}$. (D) Ureter de Sapajus apella onde é possível observar o epitélio de transição (1), tecido conjuntivo (2) e músculo liso (3). Hematoxilina Eosina. Barra: $500 \mu \mathrm{m}$. 

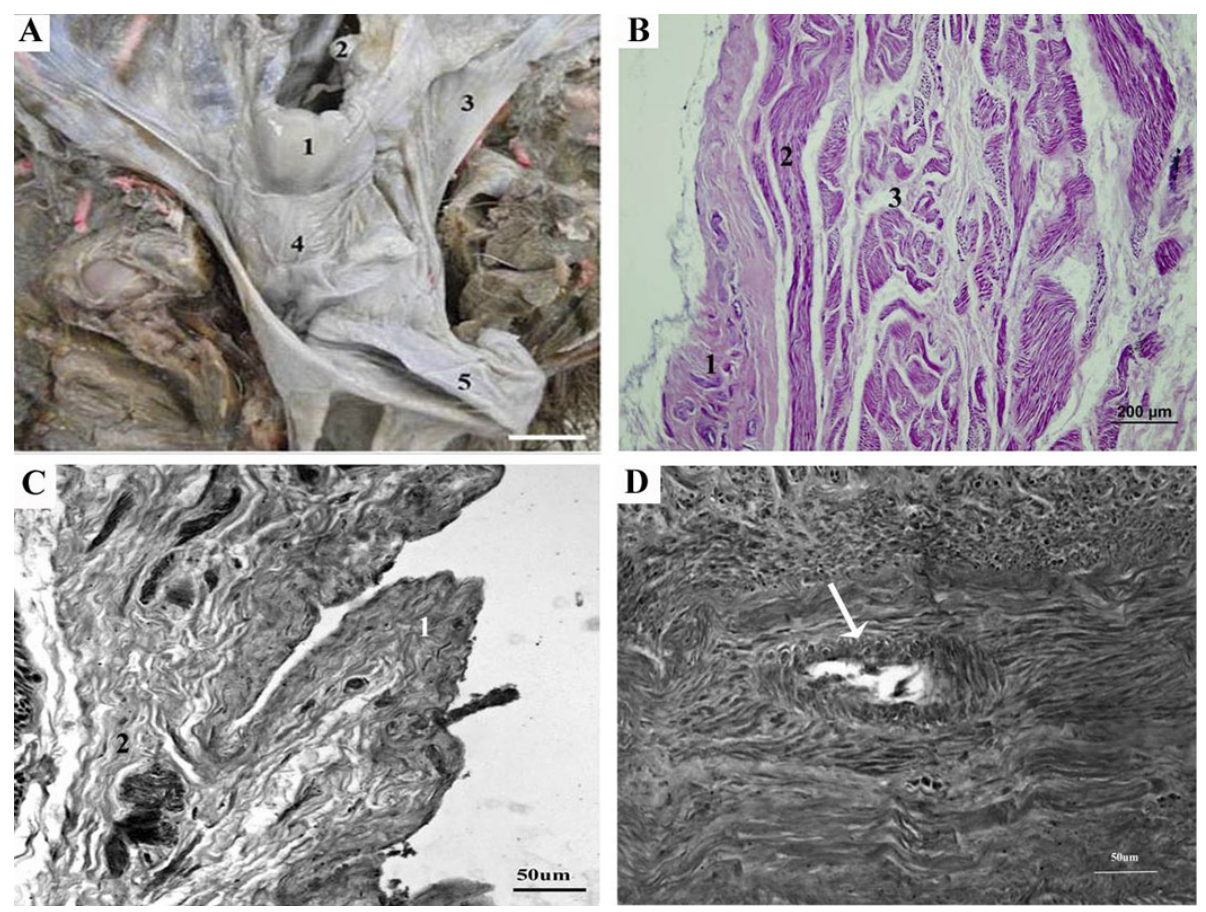

Fig.3. (A) Os órgãos genitais de Sapajus apella mostrando o útero (1), ovário esquerdo (2), ligamento largo do útero (3), cérvix (4) e bexiga urinária (5). Barra de escala: $1 \mathrm{~cm}$. (B) Bexiga urinária de Sapajus apella: Serosa (1), camada muscular longitudinal (2) e camada muscular circular (3). Hematoxilina-Eosina. Barra: 200 $\mu$ m. (C) Tecido epitelial de transição (1) e mucosa e tecido conjuntivo (2). Hematoxilina-Eosina. Barra: $50 \mu \mathrm{m}$. (D) Óstio uretral interno (seta). Hematoxilina-Eosina. Barra: $50 \mu \mathrm{m}$.

possível observar em corte transversal o óstio uretral interno (Fig.3D).

\section{DISCUSSÃO}

Os rins são glândulas de consistência firme, cor castanhoavermelhada, cujo aspecto varia consideravelmente entre os mamíferos. 0 formato mais familiar, devido ao qual foi introduzida a expressão em forma de rim ao vocábulo comum, é encontrado no cão, no gato e em pequenos ruminantes (Dyce et al. 2004).

Alves et al. (2007) afirmam que o rim direito do macaco-prego está posicionado mais cranialmente, quando comparado ao esquerdo, tal posicionamento também foi observado nos animais deste estudo. De acordo com os resultados encontrados pode-se perceber que ocorrem divergências entre autores quanto ao formato dos rins de Sapajus apella, Alves et al. (2007) descrevem que os rins desta espécie apresentam formato elíptico, enquanto Schwerk \& Nieren (1983) e Netter (1989) afirmam que apresentam formato uniformemente oval, semelhante ao observado no homem. Gaschen et al. (2000), através de dados ultra-sonográficos em $M$. fascicularis verificaram que os seus rins apresentaram diferenças em sua forma e tamanho, sendo rim esquerdo menor, mais afilado no polo cranial com o polo caudal mais largo e arredondado, já o rim direito uniformemente oval semelhante ao rim humano. Diferentemente do que encontramos para o Sapajus apella onde o rim esquerdo apresentou-se bastante semelhante ao rim humano e o rim direito apresentou-se mais afilado no polo caudal com o polo cranial mais arredondando.
Segundo Valle (2008), o rim de Callithrix jacchus ao exame ultra-sonografico apresenta aspecto ovalado, unipiramidal e a relação de ecogenicidade é igual a descrita para outras espécies de primatas. Já Miraglia et al. (1968) afirmam que os rins de $C$. jacchus possuem forma de feijão e são cobertos por uma fina cápsula fibrosa com poucas fibras elásticas. Os rins de Sapajus apella podem ser classificados como unipiramidais ou unilobares e os mesmos apresentavam-se cobertos por uma cápsula de fibrosa assim como descrito para a espécie acima. Quanto a classificação dos rins de Sapajus apella além de outros primatas não humanos estes diferem dos humanos pois este possuem rins classificados como multipapilar (Roberts 1972), uma exceção entre os primatas não humanos é o macaco aranha (Ateles geoffroyi) que possui os rins multipapilares semelhantes ao dos humanos (Goodman et al. 1977).

Histologicamente, os rins de Sapajus apella apresentam duas regiões distintas: córtex renal contendo corpúsculos renais, túbulos contorcidos proximais, distais, ductos coletores e região medular com as alças de Henle e os túbulos coletores retos culminando com a formação da pelve renal e ureter. Tal descrição é comum para espécies domésticas como o cão e o gato (Banks 1991, Dyce et al. 2004, Junqueira \& Carneiro 2008).

Na maioria das espécies domésticas, o ureter começa em uma expansão comum, a pelve renal, na qual todos os ductos papilares se abrem. Poucas diferenças na anatomia pélvica têm significado prático. A parede da pelve renal e do ureter possui uma adventícia externa, uma muscular média e uma mucosa interna (Dyce et al. 2004). Tal in- 
formação corrobora com os dados obtidos para Sapajus apella.

A bexiga urinária é um órgão distensível de estocagem e, portanto, pode não ter tamanho, posição ou relações constantes. As relações dorsais constantes da bexiga são com os órgãos reprodutivos e suas pregas de suporte, o útero e a vagina dentro do ligamento largo na fêmea e o ducto deferente dentro da prega genital no macho (Dyce et al. 2004). A bexiga de Sapajus apella estava localizada na cavidade pélvica sob o aparelho reprodutor feminino, sendo uma estrutura globosa, apresentando vértice, corpo e colo.

De maneira geral a mucosa da bexiga urinária é formada por um epitélio de transição e por uma lâmina própria de tecido conjuntivo que varia do frouxo ao denso (Junqueira \& Carneiro 2008). Em Sapajus apella a bexiga apresentou três camadas: mucosa, muscular e serosa. A túnica mucosa apresentou-se pregueada e composta por tecido conjuntivo sendo revestida por epitélio de transição que se adapta as alterações no volume. A túnica muscular é constituída por músculo liso organizado em três camadas e a túnica serosa é constituída por uma camada de epitélio que repousa sobre uma pequena quantidade de tecido conjuntivo tal descrição também é encontrada em espécies domésticas e no homem (Young \& Heath 2001, Welsch 2003, Junqueira \& Carneiro 2008).

Ultrasonograficamente, em corte sagital a vesícula urinária foi visualizada como uma estrutura globosa localizada na transição pélvico-abdominal, ventralmente ao cólon descendente e em íntima relação com o corpo do útero, em fêmeas de macaco-prego (Alves et al. 2007).

A uretra é um tubo que leva urina da bexiga para o exterior, no ato da micção. No sexo masculino, a uretra dá passagem ao esperma durante a ejaculação e, no sexo feminino, é um órgão exclusivamente do aparelho urinário (Junqueira \& Carneiro 2008).

A uretra feminina ocorre caudalmente no assoalho pélvico, abaixo do trato reprodutor. Ela passa de forma oblíqua pela parede vaginal para abrir-se ventralmente na junção da vagina e vestíbulo. Seu comprimento e sua largura variam de modo considerável entre as espécies, sendo notavelmente curta e ampla nas éguas. Em alguns animais, como na vaca e na porca, abre-se junto com o divertículo suburetral, em outros, como a cadela, em um tubérculo (Dyce et al. 2004). No Sapajus apella a uretra apresentou-se bastante curta tendo origem no trígono vesical e desembocando no vestíbulo da vagina por meio do óstio uretral externo.

Veras (2004) verificou que a uretra de Alouatta clamitans e $A$. carayase abre-se em uma papila distinta na parede ventral da vagina acima do vestíbulo. Já Pissinatti et al. (2008) relatou que em Leontopithecus a uretra surge no terço final da vagina, como um tubo muscular percorrendo a parede ventral, abrindo-se como um óstio no lúmen vaginal.

O sistema urinário de Sapajus apella apresenta características similares a outros primatas não humanos, aos humanos e aos animais domésticos. Os rins apresentaram aspectos macroscópicos semelhantes aos rins de humanos, mas com a mesma classificação de outros primatas não humanos e alguns animais domésticos. A bexiga asseme- lhou-se aos animais domésticos e aos primatas de maneira geral, humano e não humano. 0 mesmo ocorreu quando analisamos estes órgãos histologicamente.

\section{REFERÊNCIAS}

Alves F.R., Costa F.B., Arouche M.M.S., Barros A.C.E., Miglino M.A., Vulcano L.C. \& Guerra P.C. 2007. Ultrasonographic evaluation of the urinary system, liver and uterus of Cebus apella monkey. Avaliação ultra-sonográfica do sistema urinário, fígado e útero do macaco-prego, Cebus apella. Pesq. Vet. Bras. 27:377-382.

Auricchio P. 1995. Primatas do Brasil. Terra Brasilis, São Paulo. 184p.

Banks W.J. 1991. Histologia Veterinária Aplicada. 2ª ed. Manole, São Paulo. $617 p$.

Bolina R.S., Costa N.C.R., Bolina C.S.S., Fátima L.A., Silva Z. \& Barros R.A.C. 2005. Estudo anatômico macroscópico dos ossos do segmento torácico e lombar da coluna vertebral do macaco-prego (Cebus apella). Bios, Cadernos de Biologia 3:9-13.

Dyce K.M., Sack W.O. \& Wensing C.J.G. 2004. Tratado de Anatomia Veterinária. 3a ed. Elsevier, Rio de Janeiro. 813p.

Fragaszy D.M., Visalberghi E. \& Fedigan L.M. 2004. The complet capuchim: the biology of the Genus Cebus. Cambridge University Press, Cambridge. $339 p$.

Gaschen L., Menninger K. \& Schuurman H.J. 2000. Ultrasonography of the normal kidney in the cynomolgus monkey (Macaca fascicularis): morphologic and Doppler findings. J. Med. Primatol. 29:76-84.

Goodman J.R., Robert H.W. \& Roberts J.A. 1977. The unique kidney of the spider monkey (Ateles geoffroyi). J. Med. Primatol. 6:232-236.

Guerim L., Gazêta G.S., Serra-Freire N.M., Sá L.M. \& Dias J.L.C. 2001. Cebus apella (Primata: Cebidae) as a new host for Fonsecalges johnjadini (Acari: Psoroptidae, Cebalginae) with a description of anatomopathological aspects. Mem. Inst. Oswaldo Cruz 96:479-481.

International Committee on Veterinary Gross Anatomical Nomenclature 2012. Nomina Anatomica Veterinaria, $5^{\underline{a}}$ ed. Editorial Committee, Hannover, Columbia.

Junqueira L.C. \& Carneiro J. 2008. Histologia Básica. 10ª ed. Guanabara Koogan, Rio de Janeiro. 427p.

Lynch Alfaro J.W., Boubli J.P., Olson L.E., Di Fiore A., Wilson B., Gutierrez-Espeleta G.A., Chiou K.L., Schulte M., Neitzel S., Ross V., Schwochow D., Nguyen M.T.T., Farias I., Janson C.H. \& Alfaro ME. 2011. Explosive Pleistocene range expansion leads to widespread Amazonian sympatry between robust and gracile capuchin monkeys. J. Biogeography 39:272288.

Lynch A.J., Silva Jr J.S. \& Rylands A.B. 2012. How different are Robust and Gracile Capuchin Monkeys? An argument for the Use of Sapajus and Cebus. Am. J. Primatol. 74:273-286.

Miraglia T., Rossi R. \& Moreira E. 1968. Data on the kidneys of the marmoset (Callithrix jacchus). Acta Anatomica 69:274-281.

Murakami T. 1974. A revised tannic osmium method for non coated scanning electron microscope specimens. Archs Histology Japanese 36:189193.

Netter F.H. 1989. Atlas of Human Anatomy. Ciba Geigy Corp., Japan, p.567574.

Pissinatti L., Tortelly R., Porto M., Burity C.H.F. \& Pissinatti A. 2008. Morfologia macroscópica do aparelho reprodutor feminino de Leontopithecus cativos (Lesson, 1840) Primates-Callitrichidae. Arq. Bras. Med. Vet. Zootec. 60:1472-1475.

Ribeiro A.R. 2002. Estudo anatômico do plexo braquial do macaco Cebus apella: origem, composição e nervos resultantes. Dissertação de Mestrado em Anatomia dos Animais Domésticos e Silvestres, Faculdade de Medicina Veterinária e Zootecnia, Universidade de São Paulo, São Paulo. 146p.

Roberts J.A. 1972. The urinary system, p.821-840. In: Fiennes R.N. (Ed.), Pathology of Simian Primates. Vol.1. S. Karger, Basel.

Schwerk W.B. 1983. Ultraschall diagnostic, p.81-85. In: Braun B., Guenther R. \& Schwerk W.B. (Eds), Lehrbuch and Atlas. Ecomed, Alemanha. 
Teixeira D.G., Hamlett W.C., Guimarães M.A., Morini A.C., Araújo K.P., Cury F.S., Souza A.F., Vidane A.S., Ambrósio C.E. \& Miglino M.A. 2015. Morphological Tools for Describing the Male External Genitalia of Sapajus apella. Zoolog. Sci. 32(1):97-104.

Valle C.M.R. 2008. Estudo morfofuncional dos rins de primatas Callithrix jacchus em cativeiro. Tese de Doutorado em Ciências, Faculdade de Medicina Veterinária e Zootecnia, Universidade de São Paulo, São Paulo, SP. 158p.

Veras M.M. 2004. Aspectos morfológicos do aparelho reprodutor em bugios (Alouatta guariba clamitons e Alouatta caraya): o modelo feminino. Dissertação de Mestrado em Anatomia dos Animais Domésticos, Depar- tamento de Cirurgia, Faculdade de Medicina Veterinária e Zootecnia, Universidade de São Paulo, São Paulo, SP. 105p.

Watanabe I. \& Yamada E. 1983. The fine structure of lamellate nerve endings found in the rat gingiva. Archs Histology Japanese 46:173-182.

Welsch U. 2003. Sobotta, Atlas de Histologia. 6a ed. Guanabara Koogan, Rio de Janeiro. 266p.

Wilson D.E. \& Reeder D.M. 1992. Mammal species of the world: a taxonomic and geographic reference. Smithsonian Institution Press, Washington, DC. $1207 \mathrm{p}$.

Young B. \& Heath J.W. 2001. Histologia Funcional: texto e atlas. $4^{\mathrm{a}}$ ed. Guanabara Koogan, Rio de Janeiro. 415p. 